\title{
Preface to this special issue "Biologically active natural products from microorganisms and plants"
}

\author{
Hisashi Matsuda ${ }^{1}$
}

Published online: 16 June 2016

(c) The Japanese Society of Pharmacognosy and Springer Japan 2016

It is my honor to introduce this special issue of the Journal of Natural Medicines on behalf of the editorial committee. This special issue is dedicated to Dr. Satoshi Ōmura, Dr. William C. Campbell, and Dr. Youyou Tu, the winners of the 2015 Nobel Prize in Physiology or Medicine, in recognition of their outstanding contributions to the discovery of biologically active natural products over their research careers.

Dr. Satoshi Ōmura and Dr. William C. Campbell discovered avermectin, the derivatives of which have markedly lowered the incidence of river blindness and lymphatic filariasis, as well as showing efficacy against a large number of other parasitic diseases. Dr. Youyou Tu discovered artemisinin, which has significantly reduced the mortality rates for patients suffering from malaria. These discoveries based on the research of natural products have provided mankind with a powerful new means to combat debilitating diseases that affect hundreds of millions of people annually. The consequences of improved human health and reduced suffering are immeasurable.

This issue contains four invited reviews describing recent and exciting scientific advances in natural product discovery, including synthesis and stereochemistry of an antiparasitic alkaloid, artemisinin-based antimalarial research, promising chemotherapeutic candidates, and authentication of the botanical origin of herbal products.

The discovery and development of biologically active natural products has been conducted vigorously in the world and have greatly contributed to human health, including the discovery of the seed and lead compounds for modern medicines. In the past decade, approximately $40 \%$ of new drugs were developed from natural products, and research on the biologically active compounds from natural products is still very significant for the development of pharmaceutical products.

The Journal of Natural Medicines is an international journal presenting original research in naturally occurring medicines and their related foods and cosmetics. It covers the chemistry of natural products; biochemistry of medicinal plants; pharmacology of natural products and herbs, including Kampo formulas and traditional herbs; botanical anatomy; and the cultivation of medicinal plants. The journal publishes reviews, mini-reviews, original papers, notes, rapid communications, and natural resource letters.

We hope that the excellent research works published in this journal contribute to improving human health and reducing suffering.
Hisashi Matsuda

matsuda@mb.kyoto-phu.ac.jp

1 Department of Pharmacognosy, Kyoto Pharmaceutical University, Misasagi, Yamashina-ku, Kyoto 607-8412, Japan 